\title{
UM DESIGN DE AVALIAÇÃO INSTITUCIONAL
}

Narchi NZ, Friedlander MR. Um design de avaliação institucional. Rev Latino-am Enfermagem 2001 setembro-outubro; 9(5):26-31.

O objetivo deste estudo é descrever o design utilizado na avaliação de cursos técnicos de enfermagem da rede oficial de ensino do Estado de São Paulo por indicadores de desempenho institucional quanti-qualitativos. Tais indicadores, estabelecidos por egressos e mercado de trabalho, mostraram-se úteis na avaliação, constatando-se que há necessidade de acrescentar, em estudos avaliativos similares, parâmetros relacionados ao desempenho dos docentes e dos campos de ensino prático. Conclui-se que o emprego de um número razoável de indicadores permitiu a articulação entre os dados e melhor compreensão da realidade das instituições avaliadas.

PALAVRAS CHAVES: avaliação, escolas de enfermagem, ensino

\section{AN INSTITUTIONAL EVALUATION DESIGN}

This study aims at describing the design used in the evaluation of technical nursing courses in São Paulo State's Education System by means of quantitative and qualitative institutional performance indicators. Such indicators, established by former students and by the labor market, showed to be useful in the evaluation and demonstrated the need for increasing, by means of similar evaluation studies, parameters related to the academic staff's performance as well as that of practical teaching fields. It is concluded that the use of a reasonable number of indicators allowed the articulation among data and a better understanding of the evaluated institutions.

KEY WORDS: evaluation, schools, nursing, teaching

\section{UN DISEÑO DE EVALUACIÓN INSTITUCIONAL}

Este estudio tuvo como objetivo describir el diseño "desing" utilizado en la evaluación de los cursos técnicos de enfermería de la red oficial de Enseñanza del Estado de São Paulo por indicadores de desempeño institucional cuantitativos y cualitativos. Esos indicadores establecidos por egresos y mercado de trabajo, son útiles en la evaluación, constatándose la necesidad de agregar en estudios evaluativos similares, parámetros relacionados con el desempeño de los docentes y de los campos de enseñanza práctica. Se concluyó que el empleo de un número razonable de indicadores, permite la articulación entre los datos y una mejor comprensión de la realidad de las instituciones evaluadas.

PALABRAS CLAVES: evaluación, escuelas de enfermería, enseñanza

\footnotetext{
${ }^{1}$ Doutor em Enfermagem pela UNIFESP, Professor Adjunto da Graduação em Enfermagem da Universidade de Mogi das Cruzes e da Pós-Graduação em Enfermagem da Universidade do Grande ABC, e-mail: nzn@netpoint.com.br. ${ }^{2}$ Doutor e Livre-Docente em Enfermagem pela Universidade de São Paulo, Coordenadora do Curso de Graduação em Enfermagem da Universidade de Mogi das Cruzes, Orientadora da tese
} 


\section{INTRODUÇÃO}

Este estudo tem a finalidade de mostrar o percurso metodológico da tese de doutorado(1) que avaliou, por meio de indicadores de desempenho, as instituições de ensino da rede oficial de ensino do Estado de São Paulo que oferecem cursos técnicos de enfermagem. Assim, segue-se a apresentação do design* utilizado na referida avaliação institucional precedido dos aspectos teóricos que a sustentam.

São diversos os autores ${ }^{(2-9)}$ que indicam a importância do tema e ressaltam que a avaliação de instituições de ensino presta grande serviço ao identificar os aspectos de um curso que necessitam ser revistos, devendo ser utilizada com o objetivo de tomar decisões e refletir sobre as ações desenvolvidas na escola.

Nesse quadro, um processo de avaliação institucional não serve apenas à observação, ao diagnóstico de uma situação, mas essencialmente à auto-avaliação de quem vive e participa da instituição, sendo educativa na medida em que proporciona oportunidades de ação e de transformação $0^{(2)}$.

Dessa maneira, a avaliação torna-se não somente um processo de tomada de decisões como também de formação de consciência crítica, ato político intrínseco a qualquer projeto de educação séria e comprometida com a qualidade.

Esse é o sentido social da avaliação ${ }^{(10)}$, que a considera exigência de uma sociedade democrática, na medida em que faz com que as instituições prestem contas do seu desempenho no cumprimento das atribuições para as quais foram criadas e são mantidas.

A concepção fundamental é a de que as avaliações institucionais possam conduzir à transformação, de modo que as escolas cumpram seu papel na geração de conhecimentos e na solução de problemas da sociedade, o que resultará na melhoria da qualidade do processo educativo.

Qualidade em educação refere-se ao atributo diferencial, ao juízo valorativo da eficiência e eficácia, é o objeto e o objetivo do processo de avaliação ${ }^{(11)}$. Assim, julgar, examinar criticamente, fazer recomendações e criar condições para o desenvolvimento da qualidade educacional é tudo o que se pretende quando se trata de avaliação institucional.

Não existe, no entanto, consenso em relação à sua necessidade ${ }^{(12-14)}$, principalmente porque lida com juízos de valor sendo, por isso, muitas vezes refutada, constituindo-se em situação temida, na medida em que exige questionamentos e mudanças, 0 que traz como conseqüência a tendência de se manter o "status quo" e de se pensar em controle, repressão e sanção como decorrentes de processos avaliativos.

Apesar das resistências, considera-se que a partir do momento em que as avaliações forem realizadas com maior freqüência, as contestações tenderão a diminuir e os processos avaliativos deverão ser encarados como parte fundamental do desenvolvimento da própria instituição de ensino.

A própria ocorrência de falhas nesse processo deve contribuir na discussão do tema pois a avaliação tem valor sobretudo pelos questionamentos levantados no debate público, residindo aí sua importância e dimensão política ${ }^{(5)}$.

Assim, a avaliação de uma instituição escolar traduz-se na busca efetiva da melhoria de qualidade com o intuito de alcançar um produto de excelência. A avaliação do produto refere-se à determinação da eficácia global da entidade com o propósito de medir e interpretar os resultados ao término e após a conclusão do programa de um curso.

Por conseguinte, o produto da instituição escolar, o egresso, pode incorporar características positivas ou negativas, enriquecedoras ou empobrecedoras de sua personalidade, dependendo para isso do processo ensino-aprendizagem, da estrutura escolar, dos recursos físicos e materiais e do funcionamento do estabelecimento ${ }^{(15)}$.

Para realizar avaliação de instituições de ensino normalmente utilizam-se os indicadores de qualidade, ou seja, dados colhidos da realidade, que podem diferir segundo a representação ou percepção feita pelos indivíduos avaliadores nas diferentes características e perspectivas encontradas.

Por esse motivo, tais indicações devem se constituir num conjunto amplo de informações que dêem conta de apreender a essencialidade do processo, de modo a se afirmar que houve aproximação com o real ${ }^{(16)}$. Destaca-se, ainda, que entre os indicadores de qualidade, os de desempenho têm dimensão de subjetividade e implicam num julgamento de valor que varia, para melhor ou pior, de acordo com a situação encontrada na instituição de ensino ${ }^{(8,16-18)}$.

Então, na construção de indicações de qualidade de organizações complexas, como as educacionais, deve-se identificar elementos de informação que não se restrinjam aos aspectos formais ou documentais das entidades. É essa a concepção que determina a necessidade de utilizarem-se preferentemente os indicadores de desempenho nos processos avaliativos institucionais.

No caso do design da avaliação que este trabalho descreve, tais indicadores são determinados pelo produto ou resultado de um processo formativo, sendo importante ressaltar que o seu

* Concepção de um projeto ou modelo, segundo Dicionário Aurélio 
delineamento baseou-se em diversos estudos de avaliação de instituições de ensino superior ${ }^{(4,11,19-29)}$ e de ensino técnico profissionalizante ${ }^{(30-33)}$. Quanto a estas últimas, salienta-se que nenhuma é de enfermagem, sendo que o único estudo avaliativo realizado em cursos técnicos ${ }^{(34)}$ descreve diversas deficiências e levanta dúvidas sobre quais seriam suas repercussões no desempenho dos indivíduos ali formados.

A descrição do método empregado na investigação sobre a qual se baseia este artigo faz-se útil, portanto, para demonstrar como foram construídos, levantados e analisados os indicadores de desempenho institucional determinados pelo produto, no caso egressos e mercado de trabalho, em um processo de avaliação de instituições de ensino da rede oficial do Estado de São Paulo que oferecem cursos técnicos de enfermagem.

\section{O MÉTODO}

A pesquisa da qual descreve-se 0 design de avaliação instituciona ${ }^{(1)}$ adotou indicadores quanti-qualitativos de desempenho institucional relacionados aos egressos dos cursos técnicos de enfermagem e ao mercado de trabalho em que esses profissionais se inserem.

Faz-se interessante acrescentar que optou-se pela coleta quanti-qualitativa de informações por ser consensual entre os autores de referência a concepção de que a avaliação de instituições de ensino deva ser efetuada pela reunião e organização de informações quantitativas, para comparações globais e identificação de parâmetros, combinada com avaliações qualitativas que expressem a essencialidade do processo.

Tal preceito é defendido por diversos autores ${ }^{(35-38)}$, todos indicando que a articulação e complementaridade dos dados qualitativos e quantitativos contribui à compreensão mais aprofundada do que se passa no interior das escolas.

Assim, os indicadores de desempenho institucional determinados para a avaliação foram os seguintes:

- desempenho de formandos em prova de avaliação cognitiva;

- percepção de formandos e egressos quanto ao preparo profissional na instituição escolar pela qual passaram;

- percepção de enfermeiros do mercado de trabalho quanto ao desempenho dos profissionais formados pelas instituições que mantêm o curso técnico de enfermagem.

$O$ fato de serem utilizados formandos e egressos justificase por serem eles o "produto" de um processo educacional e os enfermeiros do mercado de trabalho por traduzirem-se nas pessoas que lidam diretamente com os egressos das escolas técnicas.

A coleta de dados para a avaliação obedeceu quatro etapas, nas quais levantaram-se os indicadores quanti-qualitativos já mencionados. Foram elas:

\section{Grupos de discussão com formandos}

Essa fase teve 0 objetivo de conhecer a avaliação que 0 formando faz da instituição escolar, de seu processo de formação, de como se acha preparado para o exercício da profissão.

A coleta de dados baseou-se na técnica de discussão em grupo realizada com estudantes do último bimestre letivo do $4^{\circ}$ ano do curso técnico de enfermagem, quesito determinado para que não houvesse problema de localização desses profissionais após 0 término do curso.

Durante a discussão, os formandos puderam dialogar livremente sobre a avaliação de sua formação e preparo profissional, sendo para isso empregado um formulário que norteava a conversação. Esse instrumento continha introdução, que explanava sobre a finalidade da pesquisa, e questões norteadoras, explicadas e discutidas com os grupos para facilitar sua compreensão. Após o debate livre e orientado sobre os tópicos do formulário, os dados eram registrados no instrumento pelos próprios sujeitos.

\section{Prova de avaliação de conhecimentos aplicada a formandos}

Etapa que teve a finalidade de realizar a descrição quantitativa e avaliativa das instituições, ou seja, medir a eficácia do sistema educacional profissionalizante.

Para a elaboração da prova de avaliação de conhecimentos foram escolhidos apenas testes objetivos em que os resultados alcançados não pudessem ser afetados por qualquer opinião ou julgamento de quem os corrigisse.

A confecção da prova obedeceu as fases:

- elaboração de questões baseadas em experiência profissional, em bibliografia e em provas de seleção de técnicos de enfermagem de serviços de saúde públicos e privados;

- julgamento das questões por enfermeiros responsáveis pela seleção de técnicos de enfermagem de serviços de saúde públicos e privados; - teste piloto aplicado a formandos de escolas que não se constituíram campo de estudo do trabalho.

Antes da aplicação da prova nas escolas do estudo, os coordenadores dos cursos técnicos de enfermagem, assim como os formandos, foram cientificados da finalidade da avaliação, obtendose sua colaboração e permissão.

Para que isso pudesse ocorrer, foram realizadas duas visitas às instituições no intuito de sensibilizar os formandos para a realização da prova e controlar as variáveis ansiedade e motivação no momento de sua aplicação. 
Além disso, foram dadas instruções quanto à natureza da prova, seus objetivos, tipos de questões e outros elementos esclarecedores, assim como elucidação da finalidade da pesquisa, deixando-se claro que 0 anonimato seria preservado, havendo sigilo sobre os respondentes e instituições quanto aos resultados da avaliação.

Em relação à aplicação da prova, alguns cuidados foram tomados para que não ocorressem fatores que afetassem 0 desempenho dos examinandos, especialmente os relacionados à sua orientação e ao controle de variáveis tais como condições ambientais, hora do teste, sua duração e condições físicas adequadas à acomodação.

\section{Entrevista com enfermeiros do mercado de trabalho}

A entrevista com enfermeiros teve como objetivo levantar a opinião de representantes do mercado de trabalho quanto à formação que os técnicos de enfermagem recebem.

0 instrumento utilizado para o levantamento de dados constou de um formulário com questões abertas aplicado por meio de entrevista a enfermeiros de serviços de saúde que empregavam os técnicos de enfermagem formados pelas instituições avaliadas, sendo que as falas de todos os entrevistados foram transcritas no formulário e em seguida validadas.

Nota-se que os sujeitos só poderiam ser enfermeiros, no caso, diretores de enfermagem, enfermeiros de educação continuada, enfermeiros supervisores ou assistenciais, conforme a situação do serviço de saúde escolhido, sendo essencial que se dispusessem a participar da pesquisa e tivessem contato profissional com os técnicos de enfermagem egressos das escolas do estudo.

\section{Entrevista com egressos}

Essa etapa teve o objetivo de conhecer a avaliação que 0 egresso fazia da instituição escolar pela sua percepção a respeito da preparação profissional ali recebida. Essa fase foi subseqüente à terceira, realizada no mesmo período e nos mesmos serviços de saúde, contando-se com a colaboração dos técnicos que tivessem disponibilidade e interesse em auxiliar na pesquisa.

Para coleta de dados utilizou-se a entrevista individual, semi-estruturada, com roteiro que orientou a conversação.

Os requisitos estabelecidos na seleção dos egressos que pudessem participar da pesquisa foram os seguintes: que houvessem obtido formação completa de ensino médio e profissionalizantes nas escolas avaliadas e tivessem até 36 meses de formados. Esse período foi fixado por considerar-se que após esse tempo o técnico de enfermagem, assim como qualquer outro profissional, já não disponha de elementos tão fidedignos para avaliação devido a variáveis como educação em serviço e experiência, entre outras.

A entrevista foi gravada e, nos casos em que os sujeitos não aceitaram esse recurso, foi realizada a transcrição de suas falas que depois Ihes foram lidas para que pudessem validá-las.

Após essas quatro etapas foi efetuada a análise de todos os dados. Em relação aos quantitativos, ou seja, àqueles obtidos nas provas de avaliação de conhecimento, as variáveis trabalhadas foram o número de acertos nas provas pelo número de formandos que acertaram as questões.

Quanto aos dados qualitativos, estes foram submetidos à análise temática de avaliação, tipo de análise de conteúdo usada para fazer inferências e que identifica características específicas de mensagens ${ }^{(39-40)}$. Em sua história mais recente, essa forma de analisar dados tem buscado sua lógica na interpretação qualitativa, de modo que não seja levada em conta a freqüência, mas sim, a presença ou a ausência de uma característica, ou de um conjunto delas, numa determinada mensagem.

Na busca de atingir significados no material qualitativo várias técnicas são desenvolvidas, dentre elas a análise temática, cuja unidade de registro é o tema, ou seja, uma asserção sobre determinado assunto. No caso particular do trabalho, a análise temática utilizada foi a de avaliação, definida como uma forma de exame em que se realiza o desmembramento do texto em unidades de registro de significação com objetivo específico de se ater à carga avaliativa $^{(39-40)}$

Nesse caso, apenas a dimensão das atitudes é tida em consideração e somente os enunciados que exprimem uma avaliação são submetidos à análise, sendo que a atitude caracterizou-se pelo sentido de opinião segundo um par bi-polar, ou seja, avaliação positiva ou avaliação negativa das instituições de ensino.

As etapas empregadas no processo de análise de conteúdo seguiram as descritas pela literatura ${ }^{(39-41)}$, ou seja, pré-análise, análise e interpretação e reflexão. Nessa última etapa, considerou-se que a presença de determinados temas mostravam os significados avaliativos presentes nos discursos. Os temas foram agrupados em categorias, tendo-se o cuidado de não eliminar contradições e divergências entre as falas dos diferentes sujeitos.

Após as duas etapas de análise, quantitativa e qualitativa, foi realizado o cruzamento das abordagens, ou seja, a triangulação metodológica entre as informações quantitativas e qualitativas. Essa estratégia consiste na identificação de dados por diferentes informantes em situações variadas e em momentos distintos, com a finalidade de compreender melhor o fenômeno estudado ${ }^{(41-42)}$.

Em relação aos aspectos éticos da avaliação, considera- 
se oportuno salientar que o estudo não enfrentou nenhuma resistência, mesmo havendo envolvimento de diversos sujeitos e instituições de ensino e de saúde. Recorda-se que o assunto avaliação é tema controverso e de abordagem delicada, que poderia ser rejeitado ao trazer à tona algumas deficiências.

Por esse motivo, houve o compromisso de em momento algum identificar as instituições de ensino avaliadas, havendo ainda garantia de anonimato e de sigilo de informações a todos os sujeitos que participaram da pesquisa.

Assim, os diretores das escolas investigadas e os coordenadores dos cursos técnicos de enfermagem foram informados sobre os objetivos e as etapas da pesquisa, obtendo-se autorização formal e apoio irrestrito no sentido de realizá-la. Esclarece-se, ainda, que o mesmo procedimento foi executado em relação às instituições de saúde.

No caso dos sujeitos do estudo, representados pelos formandos, egressos e enfermeiros, participaram todos os que se dispuseram livre, voluntária e conscientemente a fazê-lo, após todas as explicações e garantias de anonimato e sigilo. Tanto o consentimento informado dos indivíduos foi obtido por escrito quanto o estudo foi aprovado pelo Comitê de Ética do Programa de Pósgraduação no qual se desenvolveu.

Toda essa preocupação fundamenta-se na idéia que salienta ser a avaliação institucional assunto complicado tecnicamente, politicamente sensível e delicado, dadas suas implicações, requerendo por isso cuidados éticos e seriedade,

\section{REFERÊNCIAS BIBLIOGRÁFICAS}

1. Narchi NZ. $O$ uso de indicadores de desempenho institucional na avaliação de cursos técnicos de enfermagem. [tese]. São Paulo (SP): Departamento de Enfermagem/UNIFESP; 1999.

2. Amorim A. Avaliação institucional da universidade. São Paulo (SP): Cortez; 1992.

3. Balzan NC, Dias J Sobrinho. Avaliação institucional: teoria e experiências. São Paulo (SP): Cortez; 1995.

4. Dias J Sobrinho. Avaliação institucional, instrumento da qualidade educativa: a experiência da Unicamp. In: Balzan NC, Dias J Sobrinho. Avaliação institucional: teoria e experiências. São Paulo (SP): Cortez; 1995. p. 53-86.

5. Dias J Sobrinho. Avaliação institucional: marcos teóricos e políticos. Avaliação 1996; 1(1):15-24.

6. Durhan ER, Schwartzman S. Avaliação do ensino superior. São Paulo (SP): EDUSP; 1992.

7. Mezomo JC. Qualidade nas instituições de ensino: apoiando a qualidade total. São Paulo (SP): CEDAS; 1993. especialmente, por existir o temor de que possa desestabilizar até os indivíduos envolvidos de forma isolada.

\section{CONSIDERAÇÕES FINAIS}

Considera-se relevante salientar que o emprego dos indicadores quanti-qualitativos permitiu a articulação entre os dados e melhor compreensão da realidade das instituições de ensino avaliadas. Além disso, todas as evidências do estudo no qual empregou-se o método aqui descrito levaram a crer que um processo de avaliação não pode ser restrito, deve considerar múltiplos fatores e que o uso de um número razoável de indicadores de desempenho institucional mostrou-se essencial, pois muitos fatos se repetiram e confirmaram no decorrer de todas as avaliações.

Portanto, os indicadores empregados mostraram-se úteis na avaliação e à essa constatação acrescenta-se a necessidade de empregar, em estudos avaliativos similares, parâmetros relacionados ao desempenho dos docentes e dos campos de ensino prático, indicações não utilizadas, mas que se mostraram marcantes no decorrer dos resultados da pesquisa.

O importante é ressaltar que foi possível alcançar os objetivos propostos no trabalho ${ }^{(1)}$ do qual expôs-se apenas o método. Outros indicadores provavelmente auxiliariam a melhor compreender a realidade presente nos cursos técnicos de enfermagem, no entanto, a constatação de tais restrições transforma-se em contribuição ao demonstrar quais indicadores parecem ser mais úteis nos processos de avaliação de instituições de ensino.

8. Paul JJ, Ribeiro Z, Pilatti O. As iniciativas e as experiências de avaliação do ensino superior: balanço crítico. In: Durhan ER, Schwartzman S. Avaliação do ensino superior. São Paulo (SP): EDUSP; 1992. cap. 7, p.141-65.

9. Sguissardi V, organizadores. Avaliação universitária em questão: reformas do estado e da educação superior. Campinas (SP): Autores Associados; 1997.

10. Goldemberg J. O impacto da avaliação na universidade. In: Durhan ER, Schwartzman S. Avaliação do ensino superior. São Paulo (SP): EDUSP; 1992. p.91-112.

11. Heinzen JLN, Taglieber JE, Crema MCS, Makowiecky S. A avaliação institucional na UDESC: visualizando o fluxograma processual. Avaliação 1997; 2(1):19-24.

12. Durhan E. Avaliação, poder e democracia. Dois Pontos 1987; (38):11-2.

13. Ludke M. Por uma sociologia da avaliação. Educ Realidade 1989; 14(2):73-7. 
14. Silva RN, Davis C, Esposito YL, Mello GN. O descompromisso das políticas públicas com a qualidade do ensino. Cad Pesq 1993; (84):5-16.

15. Gonçalves EL. Avaliação de escolas médicas. Rev Hosp Clin Fac Med Univ São Paulo 1991; 46(1):41-9.

16. Trigueiro MGS. Indicadores de qualidade na universidade - um desafio para a avaliação institucional. Universidade 1994; 1(6):32030.

17. Narayanasamy A. The aplication of performance indicators to nurse education. Nurse Educ. Today 1991; 11:335-46.

18. Schwartzman J. Um sistema de indicadores para as universidades brasileiras. In: Sguissardi V (org.). Avaliação universitária em questão: reformas do estado e da educação superior. Campinas (SP): Autores Associados; 1997. p.149-75.

19. Balzan NC. A voz do estudante - sua contribuição para a deflagração de um processo de avaliação institucional. In: Balzan NC, Dias Sobrinho J. Avaliação institucional: teoria e experiências. São Paulo (SP): Cortez; 1995. p.115-47.

20. Bazzo VL, Moretti MT. Programa de avaliação institucional da Universidade Federal de Santa Catarina: fala o ex-aluno. Avaliação 1997; 2(3):67-75.

21. Belloni I, Belloni JA, Borges MM, Sobral DT. Avaliação institucional da Universidade de Brasília. In: Balzan NC, Dias Sobrinho J. Avaliação institucional: teoria e experiências. São Paulo (SP): Cortez; 1995. p. 7-113.

22. Both IJ. Avaliação institucional: agente de modernização administrativa e da educação. Avaliação 1998; 3(1):41-50.

23. Cardoso MLS, Santos OMB, Santos SMA. Avaliando o currículo de enfermagem da UFSC através do desempenho de seus egressos sob a ótica das chefias imediatas. Texto Contexto Enfermagem 1995; 4(especial):119-33.

24. Cury VAR. O currículo e a medida do social: estudo de um curso de Direito e contribuição para a avaliação institucional. In: Balzan NC, Dias J Sobrinho. Avaliação institucional: teoria e experiências. São Paulo (SP): Cortez; 1995. p.149-80.

25. Ghiorzi AR, Ribeiro EM, Fenilli RM. O currículo, o curso e a profissão, na visão de egressos da graduação em enfermagem da UFSC. Texto Contexto Enfermagem 1995; 4(especial):69-91.

26. Nascimento AC, Gressler, LA, Resende LMG. Instrumentos para coleta de dados: subsídios para avaliação institucional. Campo Grande (MT): UFMS; 1989.

27. Saupe R, Nascimento MGP. Egressos avaliam o curso de enfermagem. Texto Contexto Enfermagem 1995; 4(especial):105-18.
28. Tavares MSG, Ruffino MC, Santos BMO, Casagrande LDR. Indicadores para a reestruturação do currículo de graduação em enfermagem: expectativas dos profissionais e apreciação do currículo por ex-alunos. Rev Gaúch Enfermagem 1988; 9(1):36-42.

29. Zainko MAS. Educação superior no Brasil: a avaliação institucional como condição para seu desenvolvimento. Educ Bras 1993; 15(30):11-23.

30. Ferreira MJA. $O$ ensino técnico industrial de $2^{\circ}$ grau visto através de seus egressos: trajetória educacional e ocupacional e representações sobre a profissão de técnico industrial e sobre o mundo do trabalho de ex-alunos de escolas técnicas do município de São Paulo. [dissertação]. São Paulo (SP): Pontifícia Universidade Católica; 1990.

31. Franco MLPB. O ensino técnico agrícola do ponto de vista de seus egressos. Cad Pesq 1987; (60):15-27.

32. Kanaane R. $O$ ensino técnico-profissional e sua representação social: análise de uma escola técnica estadual do município de São Paulo. [tese]. São Paulo (SP): Instituto de Psicologia/USP; 1989.

33. Souza MG. Escola e mercado de trabalho: um estudo sobre a Escola Técnica Federal de Mato Grosso na percepção de seus alunos. [dissertação]. São Paulo (SP): Pontifícia Universidade Católica; 1993. 34. Narchi NZ. Cursos técnicos de enfermagem - avaliação da estrutura escolar, da operacionalização curricular e da disciplina Enfermagem Materno-Infantil. [dissertação]. São Paulo (SP): Escola de Enfermagem/USP; 1994.

35. Demo P. Avaliação qualitativa. $3^{\mathrm{a}}$ ed. São Paulo (SP): Cortez; 1991.

36. Dias J Sobrinho. Avaliação quantitativa, avaliação qualitativa: interação e ênfases. In: Sguissardi V, organizador. Avaliação universitária em questão: reformas do estado e da educação superior. Campinas (SP): Autores Associados; 1997. p.71-89.

37. Marques W. $O$ quantitativo e o qualitativo na pesquisa educacional. Avaliação 1997; 2(3):19-23.

38. Santos JC Filho, Gamboa SS. Pesquisa educacional: quantidadequalidade. São Paulo (SP): Cortez; 1995.

39. Bardin L. Análise de conteúdo. Lisboa (POR): Edições 70; 1977. 40. Minayo MCS. O desafio do conhecimento: pesquisa qualitativa em saúde. São Paulo (SP): Hucitec; 1992.

41. Triviños ANS. Introdução à pesquisa em ciências sociais: a pesquisa qualitativa em educação. São Paulo (SP): Atlas; 1987.

42. Koizumi MS. Fundamentos metodológicos da pesquisa em enfermagem. Rev Esc Enfermagem USP 1992; 26(especial):33-47. 